\section{Total home-cage activity as a function of the estrous cycle and wheel running in the rat ${ }^{\text {etc. }}$. \\ WILMAR A. JENNINGS* \\ Howard University, Washington, D.C. 20001}

Activity was time-sampled and examined with reference to three variables: the estrous cycle, access to running wheels, and individual differences in exploratory behavior. Total activity paralleled exploratory behavior as the latter fluctuated with the estrous cycle. When behaviors were averaged over days, running did not affect total activity. Implications for a regulation of total activity were discussed.

According to Premack \& Premack (1963), total activity or behavior output is regulated or maintained constant as specific behaviors vary. The supporting data showed that feeding decreased when rats were given access to running wheels and increased when the rats were subsequently deprived of the wheels. More recent studies, using complete recordings of activity, have demonstrated situations which not only change the probability of behaviors but also the level of total behavior. For example, Richards (1966) and Finger (1969) found that total activity fluctuates with the estrous cycle; Bolles (1965) and Mathews \& Finger (1966) found a positive relation between total activity and days of complete food deprivation.

Since Premack \& Premack (1963) recorded only feeding and running, one cannot be certain that running merely reduces other behaviors; running may also add to the level of total activity. Both the estrous cycle and food-deprivation situations involve a positive relation between exploratory behavior and total activity; it is plausible that running increased by making a running wheel available also increases total activity. The present study examined this possibility. The results have been reported in abstract form (Jennings, 1969b).

\section{SUBJECTS}

Ten adult female rats were assigned to two groups: five were given ad lib access to running wheels (the runners) and the other five were not (nonrunners). Two rats in each of the two conditions were Long-Evans hooded rats; three rats in each of the conditions were Sprague-Dawley albino rats from Sprague-Dawley, Inc. Within the two strains the rats were assigned randomly to the two conditions.

* Requests for reprints should be sent to Wilmar A. Jennings, Department of Psychology, Howard Unviersity. Washington, D.C. 20001.

\section{ENVIRONMENTAL CONDITIONS}

All of the rats were maintained in cages of hardware cloth $(10 \times 6 \times 5$ in.); the runners were additionally given ad lib access to running wheels (14 in. in diam). The rats were maintained in their respective experimental conditions for 1 month prior to observation. Ground Purina Chow in sponge dishes and water in 100-ml drinking tubes were available ad lib. During observation in the dark, a red light provided $2-3$ fc of illumination in the area of the rats. The Long-Evans hooded rats experienced a light-dark cycle of $10 \mathrm{~h}$ of dark and $14 \mathrm{~h}$ of light, and the Sprague-Dawley rats, a cycle of $12 \mathrm{~h}$ of dark and $12 \mathrm{~h}$ of light (the strains were studied in different laboratories).

\section{PROCEDURE}

Previous research (Jennings, 1969a) found that minima of feeding and maxima of running occurred in estrus as defined by the vaginal smear. To avoid pseudopregnancies, behavior rather than the smear was used to define the estrous cycle. Cycles of feeding and exploratory behavior with periods of 4-5 days were used as the criterion.

Two rats, a runner and nonrunner of the same strain, were simultaneously observed with the time-sampling procedure. The $E$ observed and classified activity once every 5 sec. The $E$ alternated between the two rats; thus, the behavior of a rat was sampled at $10-\mathrm{sec}$ intervals. The pair of rats was observed for $12 \mathrm{~h}$ a day, over 10 days; the $12 \mathrm{~h}$ were divided into two 6 - $h$ periods beginning $12 \mathrm{~h}$ apart (e.g., 6:00-12:00 p.m. and 6:00-12:00 a.m.). At the end of the 10-day period, the $\mathrm{E}$ repeated the procedure with the $12 \mathrm{~h}$ not previously sampled. Activity was classified as feeding, drinking, grooming, exploratory behavior (subclassified into running, locomotion other than running, rearing, stretching, sniffing, standing, gnawing of objects), nonactive postures (subclassified into crouching, lying on the side, and the sternoabdominal position), and miscellaneous behaviors (e.g., defecation, urination, food sifting,

(1)

RESULTS

Estrous Cycle

The rats were initially selected without regard to the presence of estrous cycles. Following an examination of the data and using behavioral criteria, the $E$ concluded that only 7 out of 10 rats showed clear evidence of an estrous cycle. Fig. 1 shows the course of behavior over 10 days in a runner with a definite estrous cycle. The ordinate represents the number of observations of a behavior over $12 \mathrm{~h}$. Feeding, drinking, grooming, exploratory behavior, and nonactivity are described. An examination of Fig. 1 shows that estrous variations occurred in exploratory behavior, feeding, and nonactivity, but not in grooming or drinking. This conclusion is supported by Pearson $r$ correlation coefficients, relating two behaviors and using a sample of 10 days. (The .05 level of significance and two-tailed tests were used throughout the present study.) The rat described in Fig. 1 showed a negative relation between exploratory behavior and nonactivity $(\mathrm{r}=-.932)$ and exploratory behavior and feeding $(r=-.748)$; but exploratory behavior was not related to grooming or drinking.

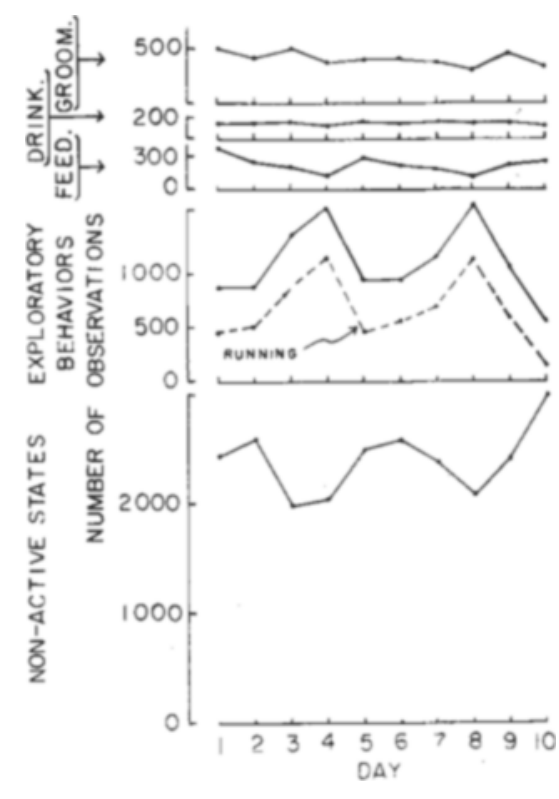

Fig. 1. Number of observations of feeding, drinking, grooming, exploratory behavior, and nonactivity in a $12 \cdot h$ period as a function of days in a rat given access to a running wheel. 
In general, the above-mentioned results were typical of the sample. Six of the seven rats showed a negative relation between exploratory behavior and nonactivity; four rats showed a negative relation between exploratory behavior and feeding; one rat showed a negative relation between grooming and exploratory behavior; and no rats showed a relation between exploratory behavior and drinking. As additional evidence, analyses of variance were used to compare behaviors on the date when exploratory behavior was maximal (estrus) with behaviors on the date when exploratory behavior was minimal (diestrus). The results showed that feeding was lower in estrus than in diestrus $(\mathrm{F}=23.317 ; \mathrm{d} f=1 / 6$; $p<.005$ ), and resting was higher in diestrus than in estrus $(F=10.668$; $\mathrm{df}=1 / 6, p<.025)$. Grooming and drinking did not differ between estrus and diestrus.

Access to a Running Wheel

The effect of running on total activity was examined. A measure of behavior over $24 \mathrm{~h}$ was obtained by averaging observations per $12 \mathrm{~h}$ over 10 days for the first 12 -h period and adding it to the average of behavior over the second $12-\mathrm{h}$ period. The complete sample of 10 rats was examined. The results showed that the runners groomed less than the nonrunners $(F=8.953 ; \quad d f=1 / 8$; $\mathrm{p}<.05)$. Although the runners showed greater feeding, drinking, and exploratory behavior than the nonrunners, none of these differences were significant. The runners and nonrunners also did not differ in non-activity, which averaged $66 \%$ of $24 \mathrm{~h}$.

\section{Individual Differences}

The relation between exploratory behavior and other behavior states was further examined with correlation coefficients comparing two different behaviors with the sample of 10 rats. The scores represented observations per $24 \mathrm{~h}$, the measure described above in the test of the effects of running wheels. The results showed that exploratory behavior was negatively related to nonactivity $(\mathrm{r}=-.871)$ and to grooming $(\mathrm{r}=-.651)$, and exploratory behavior was positively related to drinking $(r=.822)$. No relation between exploratory behavior and feeding was found.

Test-retest reliability correlation coefficients were also calculated, using three kinds of comparisons. First, observations over Days 1-5 were compared with those of Days 6.10 in the first period of observation (the sum of the first $6 \mathrm{~h}$ of each phase of the light-dark cycle). The resulting correlation coefficients were as follows: feeding, .619; exploratory behavior, .968; drinking, .995; grooming, .938; and nonactivity, .961 . Secondly, this procedure was repeated with the second 10-day period of observation (covering the hours not previously time-sampled). The resulting values were as follows: feeding, .873; exploratory beahvior, .964 ; drinking, .909; grooming, .916; and nonactivity, .872 . Thirdly, behavior averaged over all 10 days of the first period was compared with that of the 10 days of the second period of observation. The resulting values were as follows: feeding, .173 exploratory behavior, .876; drinking, .970; grooming, .759; and resting, 675. The reliability measures show that feeding was the least reliable behavior; also, observations taken from different times of the day were less reliable than observations taken from the same time of the day.

\section{DISCUSSION}

The data support the regulation of total activity (Premack \& Premack, 1963 ) in that running did not increase total activity; rather, grooming and nonrunning exploratory behaviors declined when running was added to the activity of the rat. These results, however, were based on behavior averaged over 10 days. When day-to-day variations in behaviors were compared, total activity followed the pattern of exploratory behaviors as affected by the estrous cycle. An alternative way of viewing the regulation of activity is in terms of a regulation of sleep. Colvin, Whitmoyer, \& Sawyer (1969) found that sleep, according to EEG criteria, varied with the estrous cycle in a manner parallel to nonactivity recorded in the present study. A reasonable conclusion is that the estrous cycle changes the level of total activity (or of sleep) regulated.

Premack \& Premack (1963) did not propose a specific mechanism which would account for the regulation of total activity. One possible mechanism is a competing-behavior model, in which the animal is prepared to emit a set of behaviors varying in drive strengths, only one of which can occur at a given moment. Such a model predicts a short-term inverse relation between a prepotent and a weaker behavior. In general, the data do not support such a model; for example, exploratory behavior as it fluctuated with the estrous cycle varied inversely with feeding but not with grooming or drinking. The competing-behavior model would have to explain why feeding is more likely to be inhibited by exploratory behavior than grooming or drinking. Recent studies (Wade \& Zucker, 1970) have shown that estrogen inhibits feeding independently of its effects on exploratory behaviors; thus, the relation between exploratory behavior and feeding as both fluctuate with the estrous cycle is not merely a cause-effect behavior-competition relation. Additionally, if the competing-behavior model were adequate, the long-term reduction in grooming by running would be paralleled by a short-term inverse relation between grooming and running, which was not obtained (see Fig. 1).

A more satisfactory model of the regulation of total activity might be provided by a consideration of factors which initiate drives. To the extent that the behaviors studied are controlled by external stimuli which are relatively specific, the addition of a running wheel would affect behavior not only by adding a competing behavior but also by changing the environment. For example, as the rat spends more time in the wheel, he spends less time in the adjacent cage, where some nonrunning behaviors are more likely to occur. Secondly, running may have systemic effects which in turn affect nonrunning behaviors. For example, evidence suggests that running in wheels increases adrenal secretions which affect nonrunning behaviors (Riss, Burstein, Johnson, \& Lutz, 1959).

\section{REFERENCES}

BOLLES, R. C. Effects of deprivation conditions upon the rat's home cage behavior. Journal of Comparative \& Physiological Psychology, 1965, 60 , 244-248.

COLVIN, G. B., WHITMOYER, D. I., \& SAWYER, C. H. Circadian sleep-wakefulness patterns in rats after ovariectomy and treatment with estrogen. Experimental Neurology, 1969, 25. 616-625.

FINGER, F. W. Estrus and general activity in the rat. Journal of Comparative \& Physiological Psychology, 1969, 68, 461-466.

JENNINGS, W. A. Voluntary running and the estrous variation in food intake in the rat. Psychological Reports, 1969a, 25, 199-203.

JENNINGS, W. A. Estrous variations in the total behavior output of the female rat. American Zoologist, 1969b, 9, 1067

MATHEWS, S. R., \& FINGER, F, W. Direct observation of the rat's activity during food deprivation. Physiology \& Behavior, $1966,1,85-88$

PREMACK, D. \& PREMACK, A. J. Increased eating in rats deprived of running. Journal of the Experimental Analysis of Behavior, 1963, 6, 209-212.

RICHARDS, M. P. M. Activity measured by running wheels and observation during the oestrous cycle, pregnancy and pseudopregnancy in the golden hamster. Animal Behaviour, 1966, 14, 450-458.

RISS, W., BURSTEIN, S. D., JOHNSON, R. W., \& LUTZ, A. Morphologic correlates of endocrine and running activity. Journal of Comparative \& Physiological Psy chology, 1959, 52, 618-620.

WADE, G. N., \& ZUCKER. I. Modulation of food intake and locomotor activity in female rats by diencephalic hormone implants. Journal of Comparative \& Physiological Psychology, 1970, 72, 328-336 